Research Article

\title{
Nuclear Magnetic Resonance Study on Microstructure and Permeability of Coals of Different Ranks
}

\author{
Jiajia Liu $\left(\mathbb{D},{ }^{1,2,3}\right.$ Jianmin Hu, $^{2}$ Gaini Jia, ${ }^{2}$ Jianliang Gao, ${ }^{1,2,3}$ and Dan Wang ${ }^{1,3}$ \\ ${ }^{1}$ The Collaborative Innovation Center of Coal Safety Production of Henan Province, Jiaozuo 454003, China \\ ${ }^{2}$ School of Safety Science and Engineering, Henan Polytechnic University, Jiaozuo 454003, China \\ ${ }^{3}$ State Key Laboratory Cultivation Base for Gas Geology and Gas Control, Henan Polytechnic University, Jiaozuo 454003, China
}

Correspondence should be addressed to Jiajia Liu; liujiajia@hpu.edu.cn

Received 12 September 2019; Revised 17 June 2020; Accepted 24 July 2020; Published 11 August 2020

Academic Editor: Behzad Nematollahi

Copyright ( 92020 Jiajia Liu et al. This is an open access article distributed under the Creative Commons Attribution License, which permits unrestricted use, distribution, and reproduction in any medium, provided the original work is properly cited.

The microscopic pore development of most coal seams in China leads to different permeability of coal seams and different gas drainage efficiency. Representative three coal rank coal samples were selected for saturation-centrifugation observation. The microscopic pore characteristics of coal samples were measured by nuclear magnetic resonance and liquid nitrogen adsorption methods. The experimental results showed that the coal samples were subjected to saturation-centrifugation and nuclear magnetic resonance (NMR) tests. It was found that the pores of the low-rank coal (XJ-1, XJ-2) were developed at various stages, and the connectivity between the pores was good and the permeability was also good. The adsorption pores of the intermediate coal (HB-1, HB-2) and high-rank coal (ZM-1, ZM-2) were relatively developed, and the connectivity between the pores was slightly poor. The parallel coal seam samples of coals of different ranks were better than the vertical bedding. The adsorption of liquid nitrogen showed that the low-order coal had more open pores and good gas permeability; the high-order coal had more openings at one end, more ink bottles, and narrow holes, and the gas permeability was not good. Studying the micropore structure and permeability of coals of different ranks has guiding significance for mastering the law of coal seam gas storage and transportation, extracting drilling arrangements, and increasing gas drainage and reducing greenhouse effect.

\section{Introduction}

Coalbed methane is a hydrocarbon gas that is present in the coal seam and contains gas as the main component, about $80 \% \sim 90 \%$ in the adsorbed state on the surface of the coal matrix particles and $10 \% \sim 20 \%$ in the pores and cracks of the coal body. It is an unconventional natural gas produced with the formation of coal, and it is also a clean, high-energy highquality energy source. It is commonly known as "gas" in mines, with high calorific value and no pollution after combustion [1-4]. When the concentration of coalbed methane is $5 \% \sim 16 \%$, an explosion will occur in the event of an open flame, and the power is extremely destructive. This is the root cause of coal mine gas explosion. Direct discharge into the atmosphere produces a strong greenhouse effect, about 20 times that of carbon dioxide. If coalbed methane is first mined before coal mining, the incidence of gas explosion can be reduced, clean energy can be obtained, and the greenhouse effect can be reduced [5-7].

Coal is a kind of porous medium. The adsorption gas of coal is related to the surface area of coal, and the surface area of coal is related to the pore characteristics of coal. Therefore, Qi and Huang thought that the pore characteristics of coal bodies played an important role in adsorbing gas $[8,9]$. The pore structure and pore size distribution of coal affect the adsorption and permeability of coal $[10,11]$. However, Lu et al. thought that the pore characteristics of different metamorphic coals were different, and the degree of geological damage and the influence of geostress make coal development contain different bedding structures $[12,13]$. Therefore, it is necessary to consider the microstructure of coals of different ranks and the permeability of coal in the bedding direction. It provides a theoretical basis for the storage and 
transportation of coal seam gas and the drainage arrangement of gas drainage to improve the extraction effect.

In the past, many scholars had studied the pore and fracture structure characteristics of coal. There are direct observations [14] and indirect acquisitions [15] from research methods. Direct observation: the development of coal surface cracks was observed with the naked eye or the coal pores, crack development, and filling were observed by optical microscopy and scanning electron microscopy. Indirect acquisition: the adsorption amount was determined by physical adsorption method under a series of different relative pressures, and the adsorption curve was obtained, and the curve processing and analysis were studied [16]. Although the conventional method was widely used, it had certain deficiencies. The information of the pore fracture observed by the direct observation method was limited, and the mercury intrusion method cannot avoid the occurrence of the elastic compression effect of high pressure on coal, when $[17,18]$ tested. In general, [19] studied that conventional methods had certain limitations on the integrity and in situ properties of coal pores and fractures. Low-field NMR technology can make up for the shortcomings of conventional methods by virtue of its fast and nondestructive characteristics and adopts conventional and classic methods [20-22]. The liquid nitrogen adsorption method was used for comparative analysis.

Based on this, the focus of this study was to select three typical coal samples of different coal ranks, to test the pore structure of coal after saturating and centrifuging coal by nuclear magnetic resonance technology, and to grasp the pore development and permeability of different coal ranks. The pore characteristics of different coal structure coals were further studied by conventional method-low temperature liquid nitrogen method. Studying the pore characteristics of different coal structure coals can help to understand the differences of coal reservoirs caused by different coal structure and provide valuable data for coal mine gas disaster prevention and evaluation and development of coalbed methane resources in different coal structure development areas.

\section{Experimental}

2.1. Principle of $\mathrm{NMR} \mathrm{T}_{2}$ Analysis. Nuclear magnetic resonance (NMR) is a phenomenon in which hydrogen nuclei are aligned under the action of an external magnetic field, and resonance relaxation occurs through the superimposed external magnetic field to detect hydrogen (hydrogen, methane, etc.) fluid in coal rock mass. Relaxation characteristics: NMR study of the pores and fractures of coal rock mass is obtained by studying the relationship between nuclear magnetic resonance intensity and $T_{2}$ relaxation time to study the pores, fracture structure distribution, and size connectivity of coal and rock mass. The principle [23-27] is

$$
\begin{aligned}
\frac{1}{T_{2}} & =\rho \frac{S}{V}=F_{S} \frac{\rho}{r}, \\
r & =C T_{2} .
\end{aligned}
$$

In the above-given formula, $\rho$ is the coal surface relaxation rate, which is a parameter for characterizing the rock properties; $S / V$ is the specific surface area of the pores in the coal; $F_{S}$ is the pore shape factor (where the pores of the ball row are 3 and the plate shape and the tubular shape are all 2); $r$ is the pore radius; and $C$ is the conversion coefficient.

It can be seen from (1) that the transverse relaxation time $T_{2}$ is proportional to the pore radius. According to different relaxation mechanisms, the $T_{2}$ of different pores and cracks are different, and the positions in the $T_{2}$ spectrum are different. The smaller the $T_{2}$ is, the smaller the pore radius is represented; the area enclosed by the $T_{2}$ spectrum curve and the $X$-axis indicates the number of pores in the pore range; the number of $T_{2}$ peaks represents the development of pores; the trend change of the $T_{2}$ spectrum represents the quality of connectivity between different pores [28-30].

2.2. Nuclear Magnetic Resonance Experimental Setup. The nuclear magnetic resonance experimental instrument is a Meso-MR23-060H-I low field nuclear magnetic resonance instrument (in Figure 1) produced by Shanghai Niumag Corporation. The experimental conditions are as follows: magnetic field strength is $0.5 \mathrm{~T}, \mathrm{H}$ proton resonance frequency and RF pulse frequency are $21.67 \mathrm{MHz}$, and the magnet temperature is controlled at $32 \pm 0.1^{\circ} \mathrm{C}$.

2.3. Coal Sample Preparation and Treatment. Coal samples were used for nuclear magnetic resonance experiments. The samples were collected from the low-rank coal of the Aiweiergou mine in Xinjiang Uygur Autonomous Region, the middle-rank coal of the Hebi mine in Henan, and the highrank coal of the Zhongma mine in Jiaozuo, Henan. Fresh large samples were collected and transported back to the laboratory, and a cylindrical sample of $\varphi 25 \times 50 \mathrm{~mm}$ was prepared by parallel coal layer drilling and vertical bedding. The numbers were (XJ-1, XJ-2, HB-1, HB-2, ZM-1, and ZM-2, where 1 represented a parallel layer coal sample and 2 represented a vertical bedding coal sample). And a part of the coal pieces remaining after the core was taken in accordance with (GB/ T212-2008) "Industrial Analysis Method of Coal" for industrial analysis and testing. A certain amount of 60 80 mesh coal powder was prepared to prepare for the subsequent liquid nitrogen adsorption experiment. The basic parameters of coal samples and industrial analysis results are shown in Table 1.

First, the cylindrical coal sample prepared above was placed in an electrothermal blowing dry box (Figure 2) to be dried for 2 hours. Then, we used a vacuum saturation device (Figure 3 ) (vacuum pressure was $0.1 \mathrm{MPa}$, pumping time $12 \mathrm{~h}$ ) and vacuum saturated water treatment, and then we added distilled water exceeding the height of the coal sample to the sample every day, soaking for $48 \mathrm{~h}$, until the weight of the coal sample no longer increased, and the coal sample got saturated.

2.4. Calibration Instrument. Open the computer and NMR core measurement software, select the FID sequence and the corresponding coil (experimentally select $25 \mathrm{~mm}$ coil), quickly turn on the RF switch, put the standard oil sample into the $25 \mathrm{~mm}$ coil and set the relevant experimental 


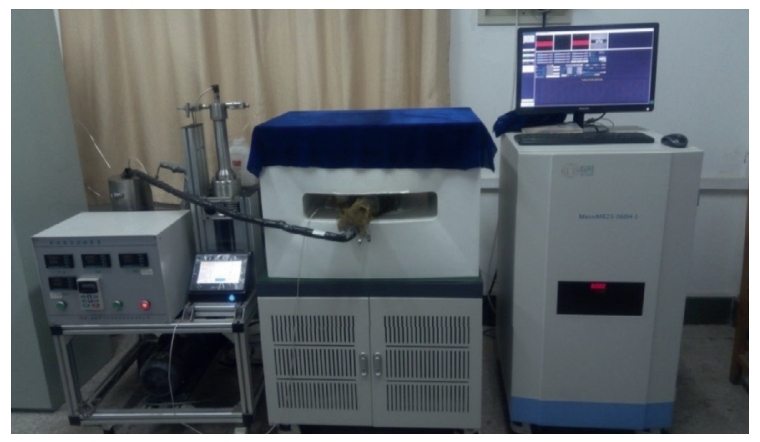

Figure 1: The physical map experimental system.

TABLE 1: Coal basic parameter test.

\begin{tabular}{lcccc}
\hline Sample & Ad (\%) & Mad (\%) & Vdaf (\%) & $R_{0} \max$ \\
\hline XJ & 7.33 & 1.02 & 40.64 & 0.78 \\
HB & 7.52 & 0.88 & 10.35 & 2.18 \\
ZM & 8.41 & 2.94 & 5.50 & 3.41 \\
\hline
\end{tabular}

parameters (see Table 2), and follow the instructions for calibration (Figure 4).

2.5. Obtaining the Rule of the Line Equation. The porosity standards were placed in the coils in the order of $0 \%, 1 \%, 3 \%$, $6 \%, 10 \%$, and $15 \%$, and the relevant parameters (signal amplitude) were tested. The nuclear magnetic resonance core analysis software automatically generated a porosity standard as shown in Figure 5.

2.6. Coal Sample Test. Put the coal sample to be tested into the center of MesoMR $\times 25 \mathrm{~mm}$ coil, open the NMR core measurement software, select the CPMG sequence, set the parameters, the specific parameters are shown in Table 3, select the established porosity mark, and test the coal sample (nuclear magnetic porosity, permeability, etc.).

2.7. NMR and Core Measurement Experiments. First, the abovementioned treated saturated coal samples were wrapped with plastic wrap (to prevent evaporation of water and reduce experimental error) and placed in a nuclear magnetic resonance apparatus. First, the nuclear magnetic resonance $T_{2}$ spectrum of the coal samples was measured, and each sample was continuously tested twice. Then the core measurement software was opened for nuclear magnetic resonance core measurement and the relevant experimental parameters were recorded. To complete the NMR experiment of saturated water sample, [31] took out the coal sample and centrifuged it in a centrifuge (Figure 6) at $25^{\circ} \mathrm{C}$ for $1 \mathrm{~h}$, and the centrifugal force was $1.38 \mathrm{MPa}$ (related research shows that the coal body had the best centrifugal effect under the centrifugal force of $1.38 \mathrm{MPa}$ ). Again the above test was carried out.

\section{NMR Experimental Results and Analysis}

The nuclear magnetic resonance $T_{2}$ spectrum of the coal sample measured under saturated and centrifuged conditions is shown in Figure 7.

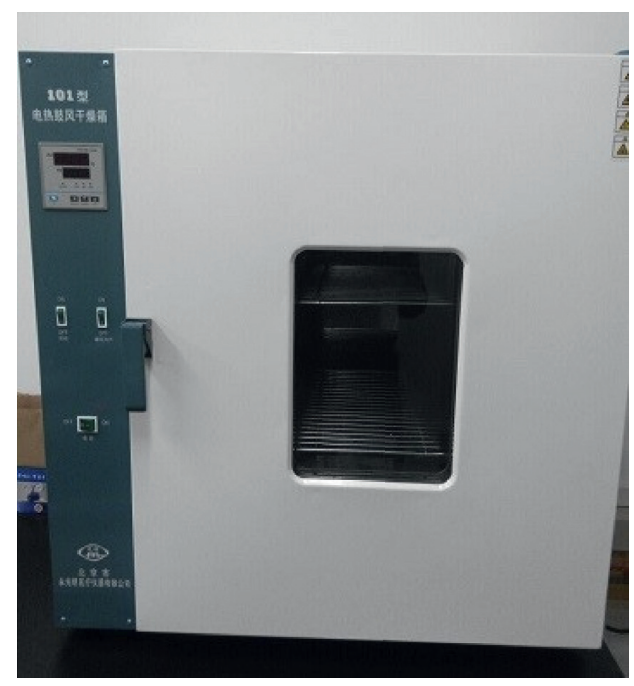

Figure 2: The electrothermal blowing dry box.

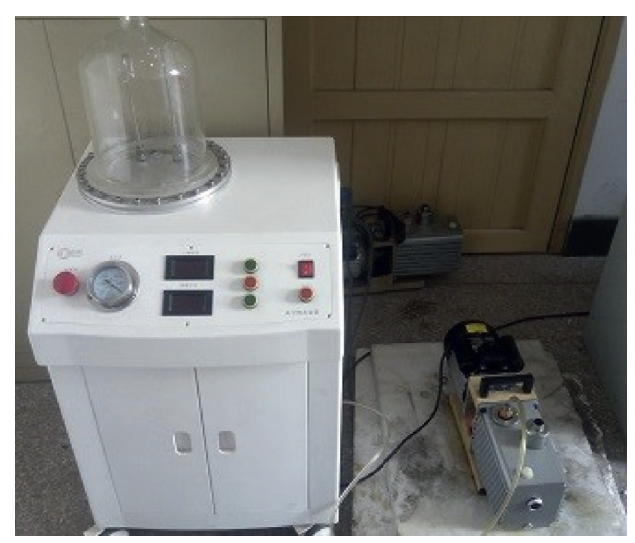

FIgURe 3: The vacuum saturation device.

TABLE 2: FID sequence parameters.

\begin{tabular}{lc}
\hline Parameter & Value \\
\hline SW $(\mathrm{kHz})$ & 100 \\
DRG & 3 \\
SF $(\mathrm{MHz})$ & 21 \\
DR & 1 \\
O1 $(\mathrm{Hz})$ & 675098.01 \\
NS & 2 \\
PRG & 1 \\
RG1 (dB) & 10.0 \\
RFD (ms) & 0.002 \\
TW (ms) & 1500.000 \\
\hline
\end{tabular}

Determine the $T_{2}$ cutoff value $\left(T_{2 c}\right)$ by NMR core measurement software to further obtain the mobility of the movable fluid and the bound fluid in Figure 8.

The authors of $[32,33]$ used $T_{2}$ cutoff value to determine the boundary between the movable fluid and the bound fluid of the coal sample and distinguished the movable fluid from the bound fluid to further judge the permeability of the coal rock mass. Theoretical general research suggested that the 


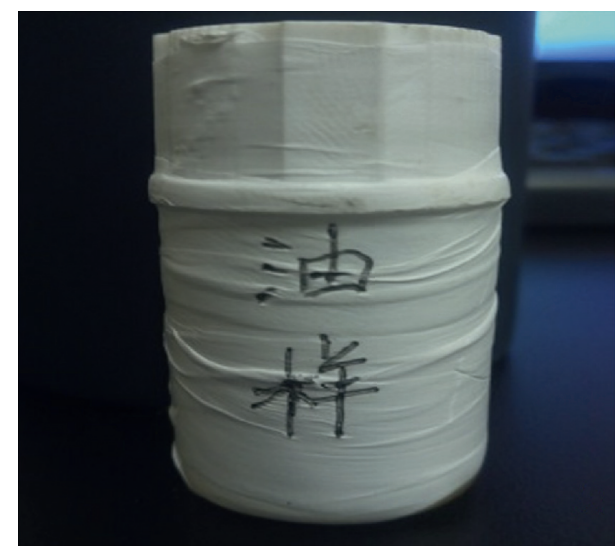

(a)

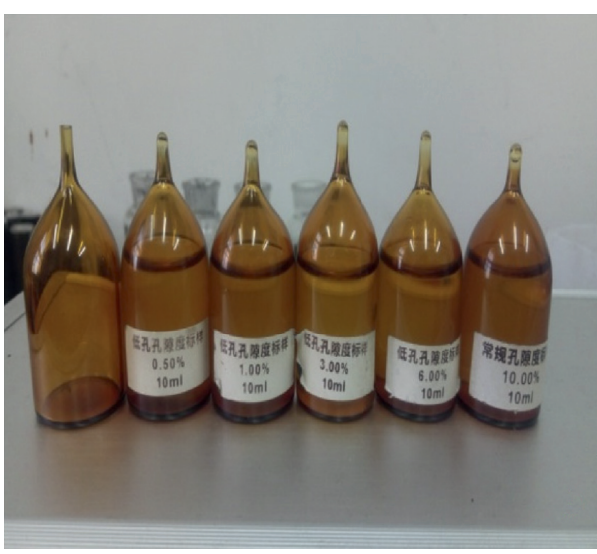

(b)

Figure 4: The experimental standard sample. (a) Oil sample. (b) Porosity standard.

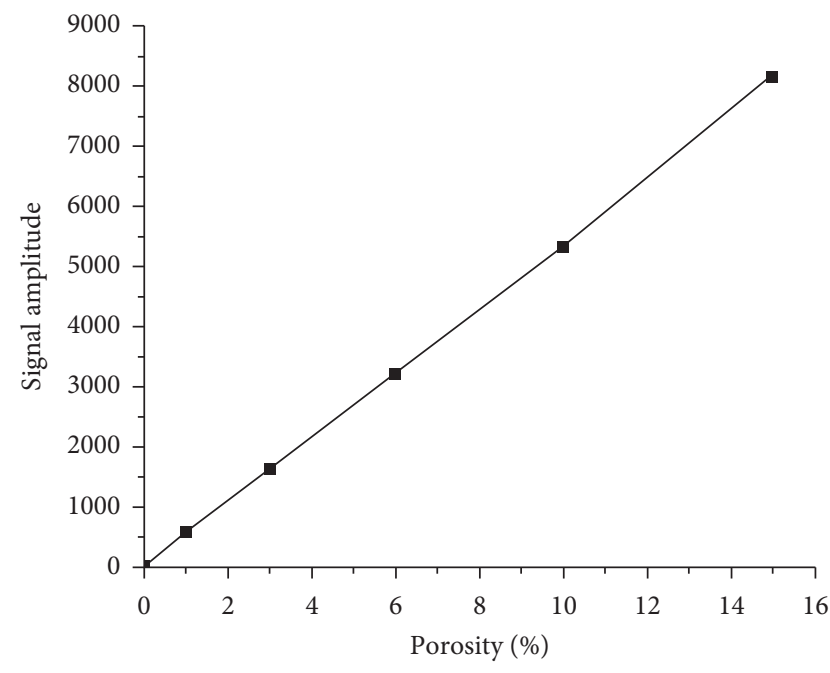

FIGURE 5: The porosity marking.

left side of the $T_{2 c}$ point was the bound fluid, which was present in the adsorption pores of the coal, and the fluid in the pores was not scooped out under the optimal centrifugal force. The right side of the $T_{2 c}$ point was a movable fluid, which meant that the fluid in the seepage hole (medium or large hole and crack) was easily ejected under the action of centrifugal force. The $T_{2 c}$ point was determined by the method of accumulating the porosity of the coal sample before and after being centrifuged, paralleling the $X$-axis from the maximum value of the $T_{2}$ spectrum integral curve after centrifugation, and prolonging the cumulative pores of the parallel line and the saturated coal sample. The rate curve intersected with one point. Starting from the intersection point, the line was perpendicular to the $X$-axis. The value of the abscissa corresponding to this line was $T_{2 \mathrm{c}}$. The $T_{2}$ cutoff values of the three coal samples are significantly different.

Since the intensity of the nuclear magnetic signal was proportional to the number of hydrogen nuclei in the sample, the porosity of the coal sample could be determined according to the nuclear magnetic resonance $T_{2}$ spectrum. Here, the irreducible porosity (IP) corresponds to the bound
TABLE 3: CPMG sequence parameters.

\begin{tabular}{lc}
\hline Parameter & Value \\
\hline SW $(\mathrm{kHz})$ & 333.333 \\
SF $(\mathrm{MHz})$ & 21 \\
O1 $(\mathrm{Hz})$ & 675098.01 \\
RFD $(\mathrm{ms})$ & 0.080 \\
RG1 $(\mathrm{dB})$ & 15 \\
P1 $(\mu \mathrm{s})$ & 23.00 \\
DRG & 3 \\
DR & 1 \\
TW $(\mathrm{ms})$ & 1500.000 \\
NS & 32 \\
PRG & 1 \\
P2 $(\mu \mathrm{s})$ & 44.00 \\
TE $(\mathrm{ms})$ & 0.202 \\
NECH & 10000 \\
\hline
\end{tabular}

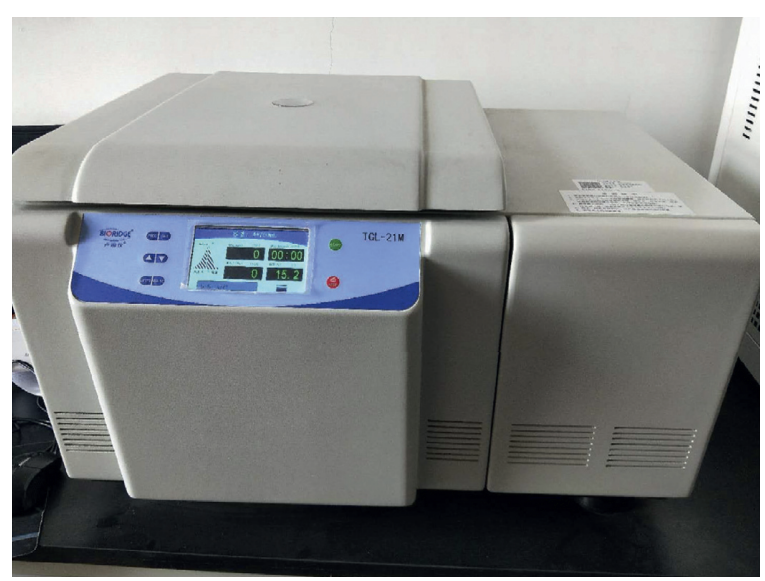

Figure 6: Centrifuge.

water fraction, and the producible porosity $(\mathrm{PP})$ corresponds to free water fraction. The $\varphi_{\mathrm{NI}}$ and $\varphi_{\mathrm{NP}}$ could be determined by comparing the $T_{2}$ spectrum under water conditions with that in centrifuged conditions for the same coal sample. The $\varphi_{\mathrm{NI}}$ and $\varphi_{\mathrm{NP}}$ were expressed by the following formula $[33,34]$ : 


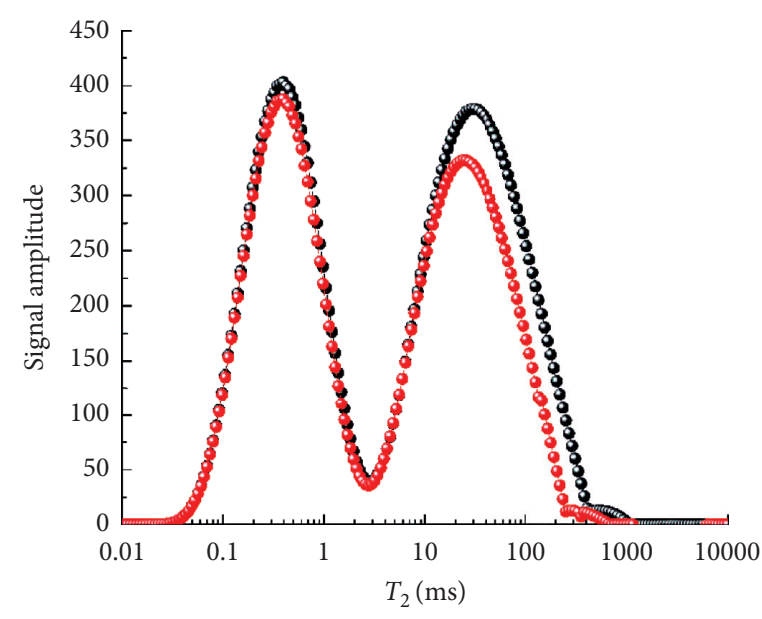

$\rightarrow T_{2}$ under saturated conditions

- $T_{2}$ under centrifuged conditions

(a)

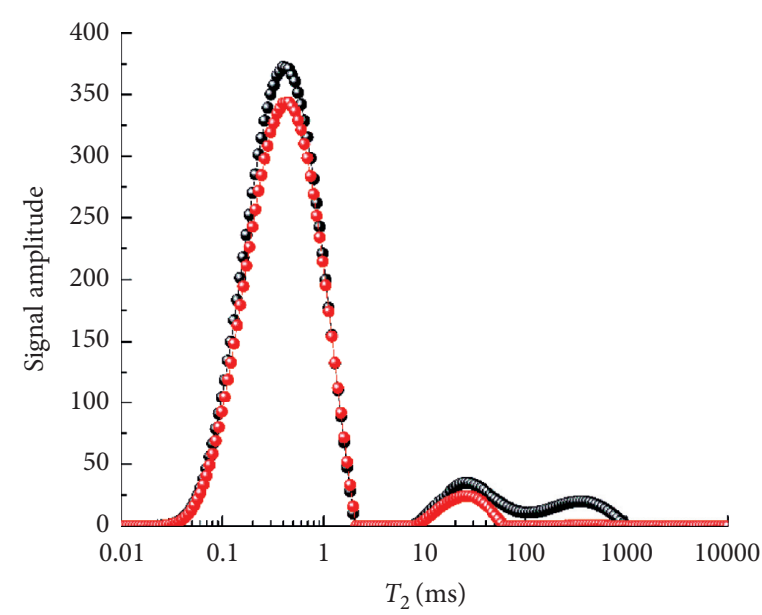

- $T_{2}$ under saturated conditions

$\multimap T_{2}$ under centrifuged conditions

(c)

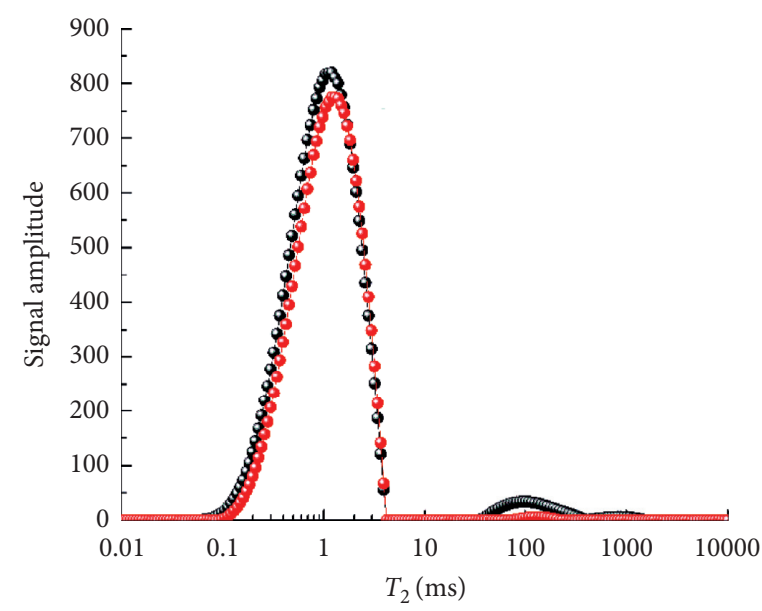

$\rightarrow T_{2}$ under saturated conditions

$\multimap T_{2}$ under centrifuged conditions

(e)

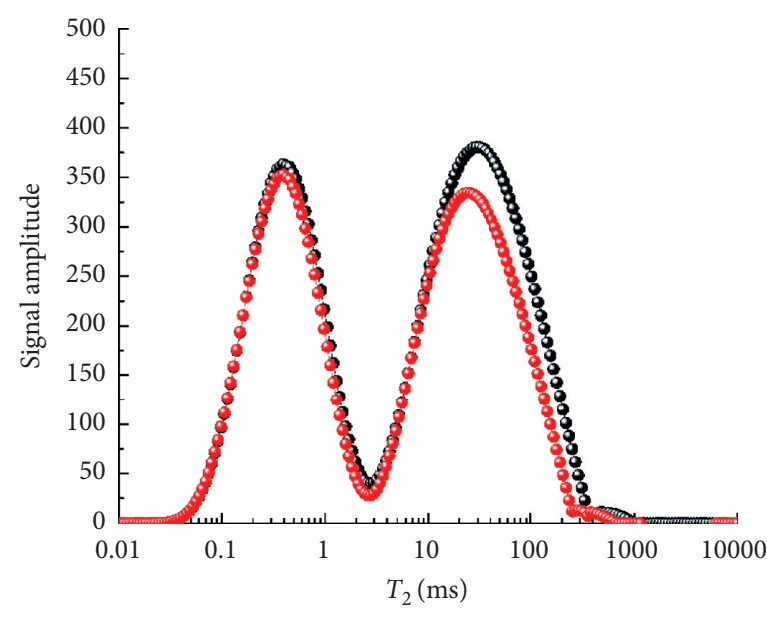

$\multimap T_{2}$ under saturated water conditions

- $T_{2}$ under centrifuged conditions

(b)

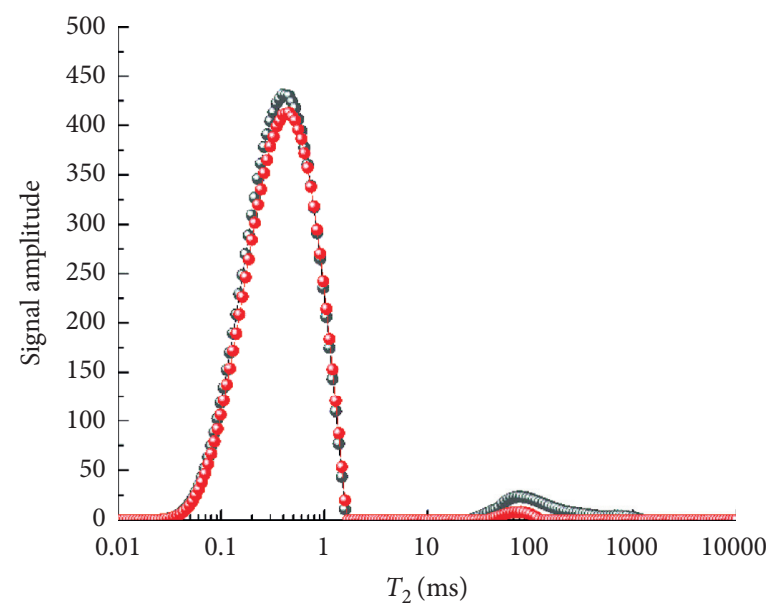

- $T_{2}$ under saturated conditions

$\rightarrow T_{2}$ under centrifuged conditions

(d)

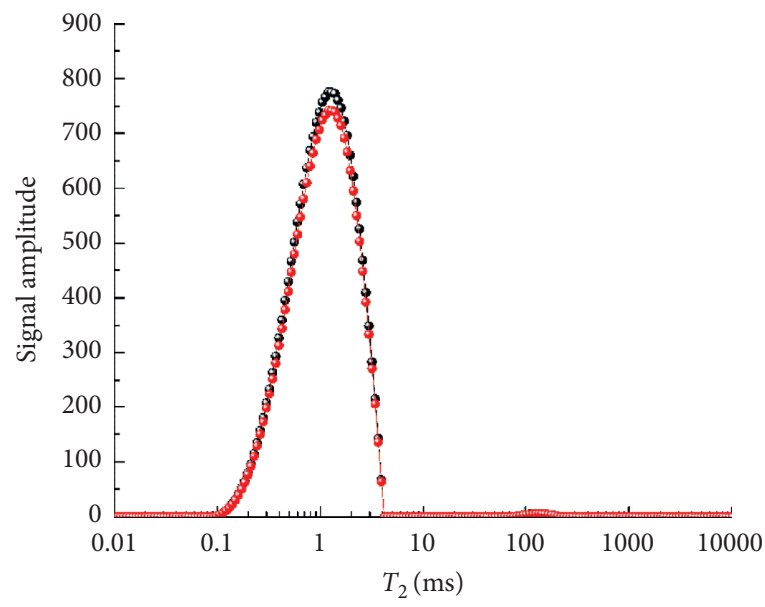

$\rightarrow T_{2}$ under saturated water conditions
$\rightarrow-T_{2}$ under centrifuged conditions

(f)

Figure 7: The $T_{2}$ spectra of coal samples with parallel and vertical layering. (a) XJ-1, (b) XJ-2, (c) HB-1, (d) HB-2, (e) ZM-1, and (f) ZM-2. 


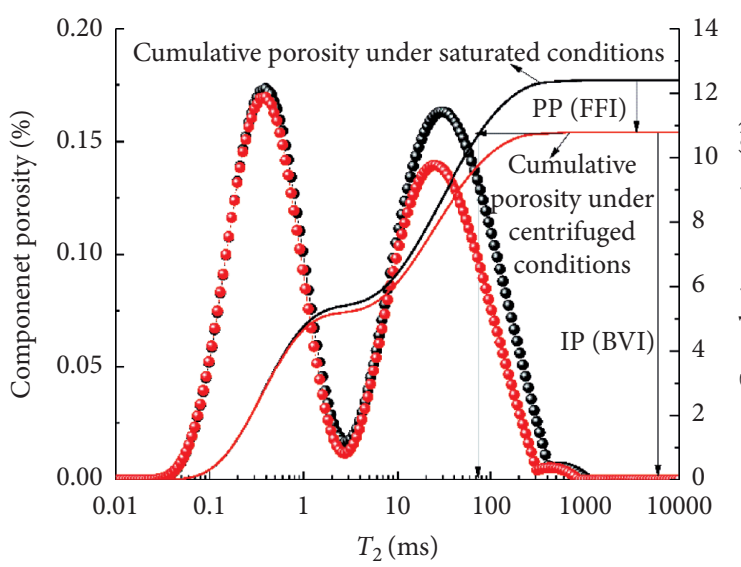

- $T_{2}$ under saturated water conditions $\multimap T_{2}$ under centrifuged conditions

(a)

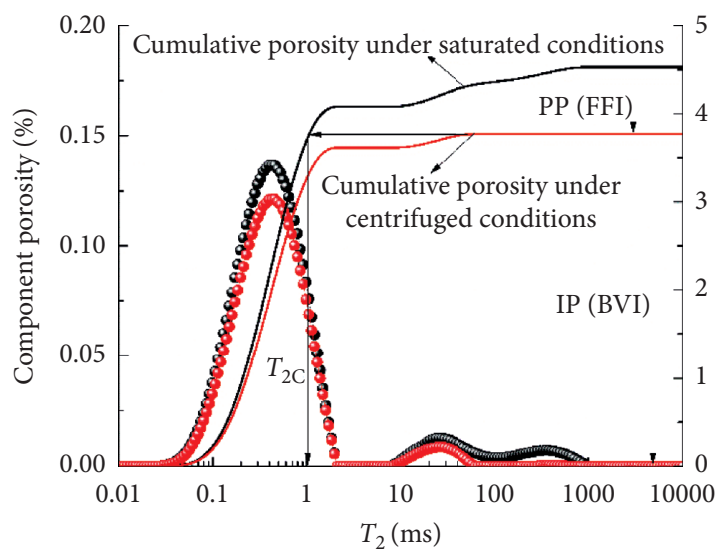

$\rightarrow T_{2}$ under saturated conditions

- $T_{2}$ under centrifugation conditions

(c)

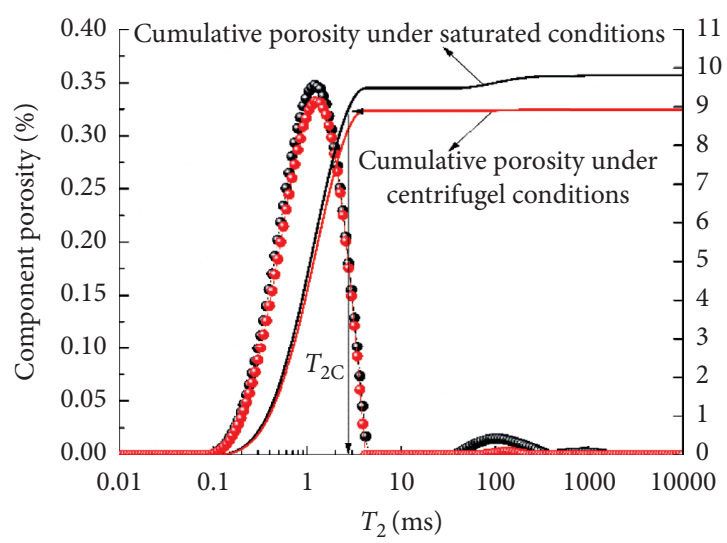

$\rightarrow T_{2}$ under saturated conditions

- $T_{2}$ under centrifugation conditions

(e)

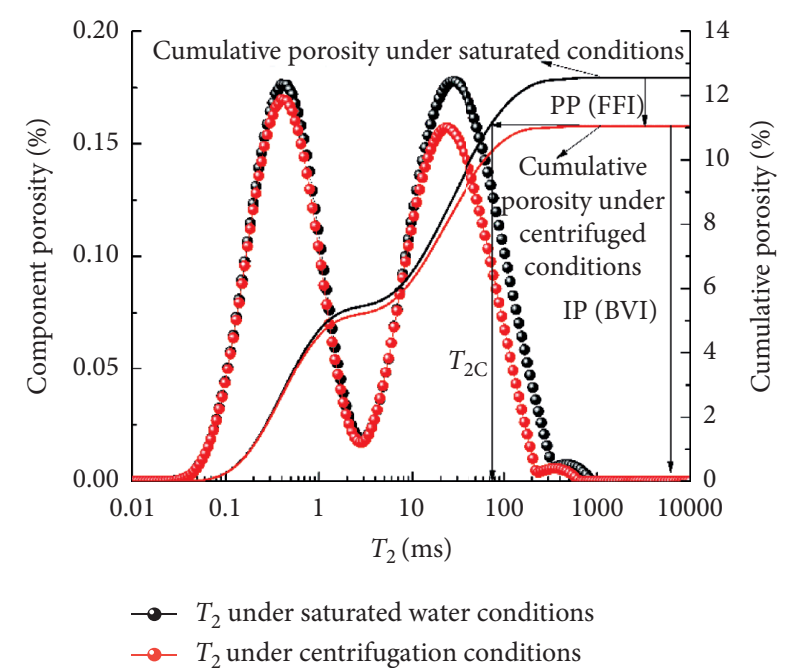

(b)

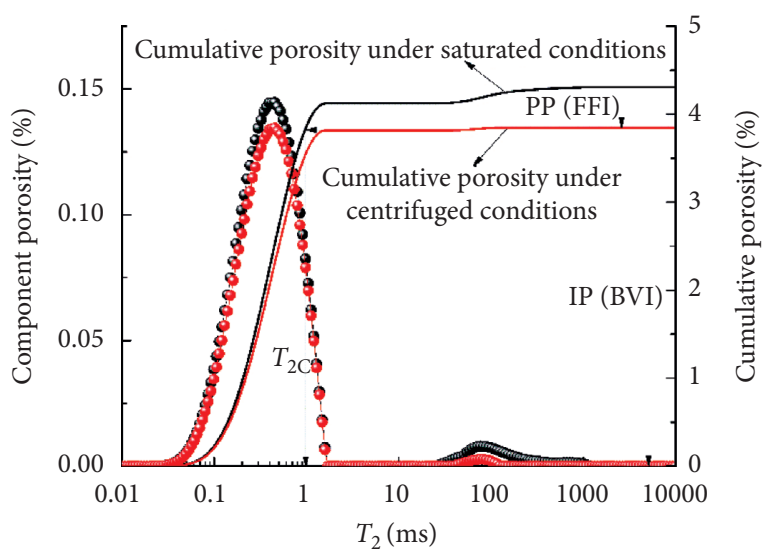

$\rightarrow T_{2}$ under saturated water conditions

- $T_{2}$ under centrifugation conditions

(d)

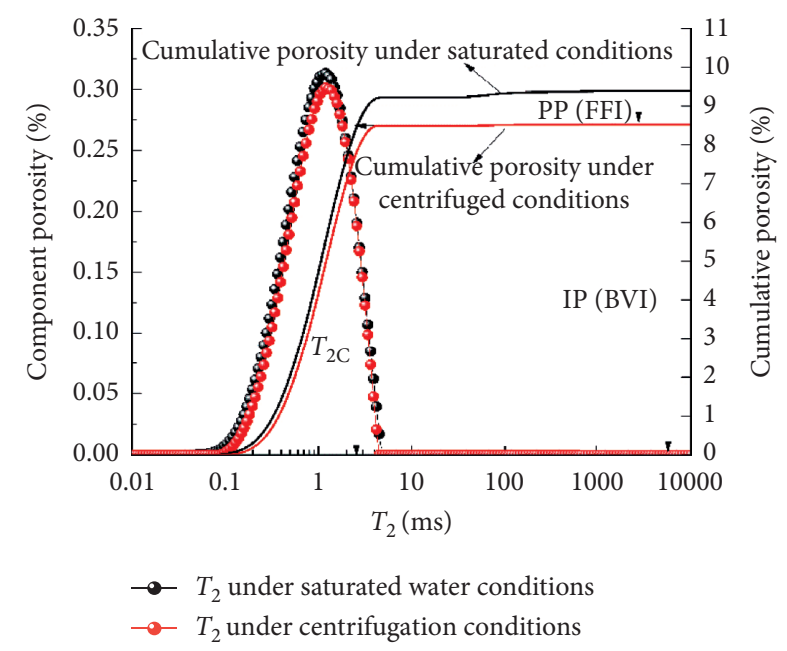

(f)

FIgure 8: Movable fluid porosity and bound fluid porosity, showing the method to calculate $T_{2 c}$. (a) XJ-1, (b) XJ-2, (c) HB-1, (d) HB-2, (e) ZM-1, and (f) ZM-2. 


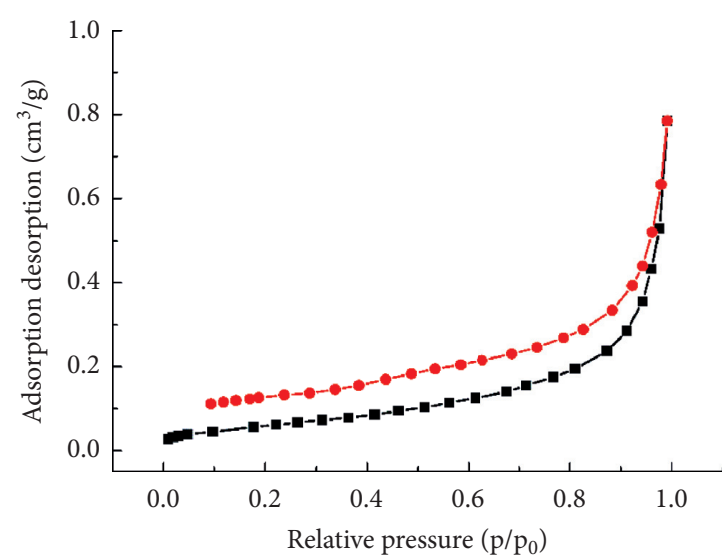

$\rightarrow$ Adsorption

$\longrightarrow$ Desorption

(a)

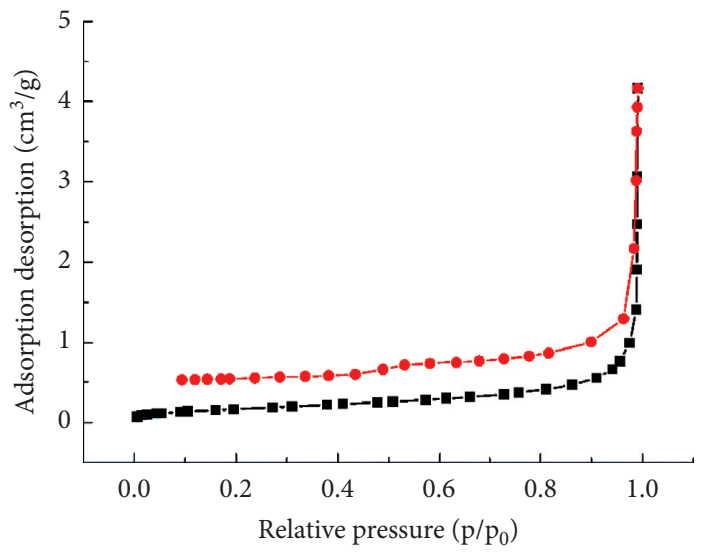

- Adsorption

$\longrightarrow$ Desorption

(c)

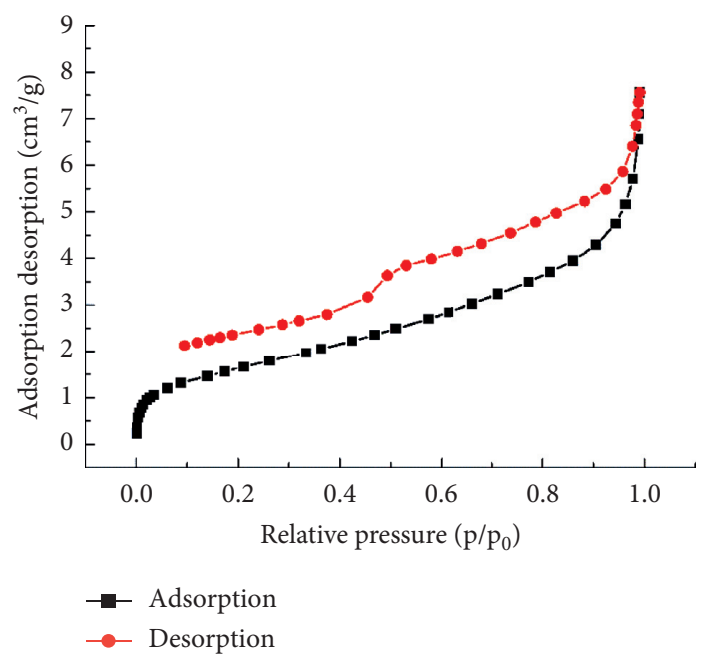

(e)

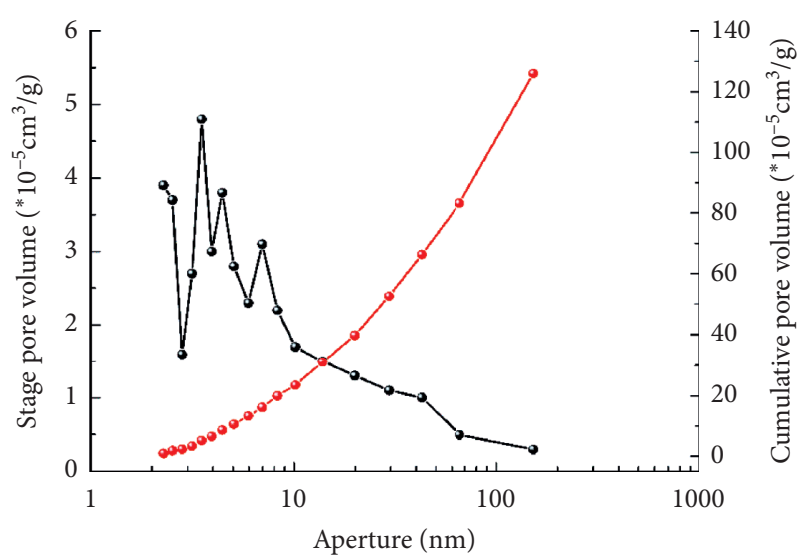

$\rightarrow \mathrm{XJ}-1$ pore volume distribution

$\rightarrow$ XJ-1 cumulative pore volume

(b)

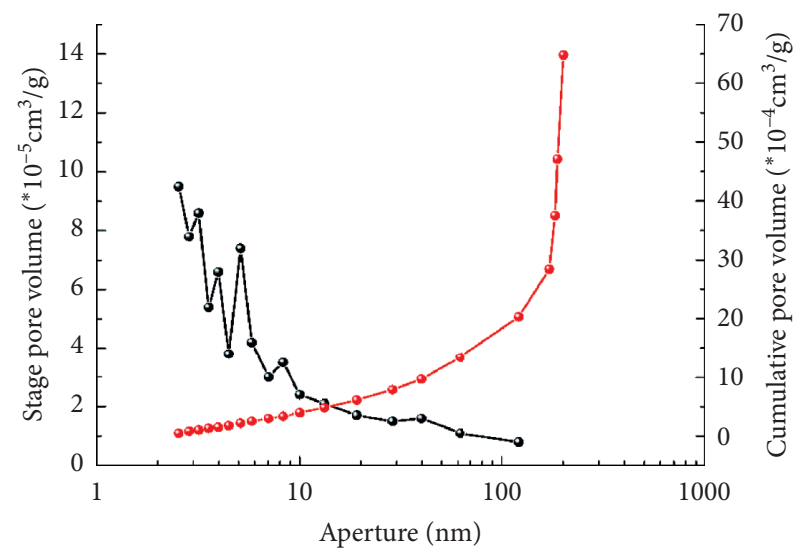

$\rightarrow$ HB-1 pore volume distribution

- HB-1 cumulative pore volume

(d)

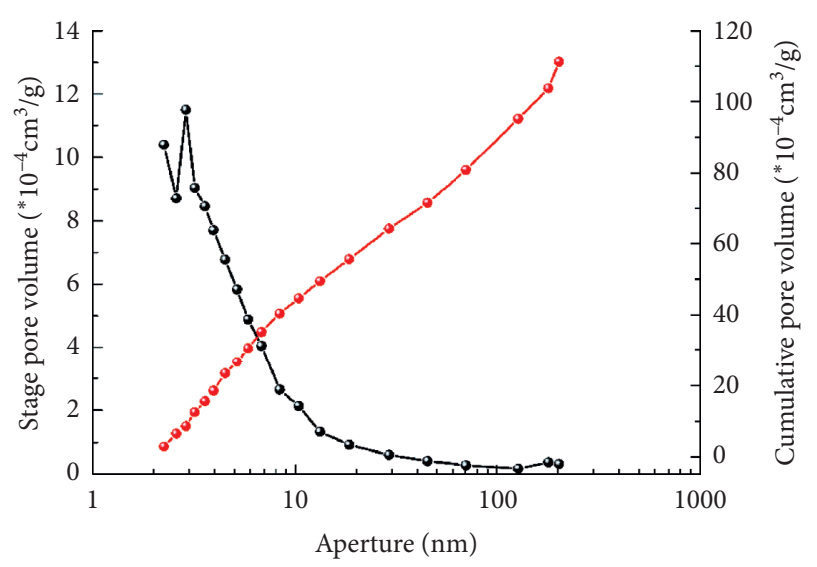

- ZM-1 pore volume distribution

$\multimap$ ZM-1 cumulative pore volume

(f)

FIgURe 9: Low temperature liquid nitrogen adsorption curve of coal sample and pore size distribution curve of coal sample. (a) XJ-1, (b) XJ2, (c) HB-1, (d) HB-2, (e) ZM-1, and (f) ZM-2. 


$$
\begin{aligned}
& \varphi_{\mathrm{NI}}=\frac{\varphi_{\mathrm{N}} \times \mathrm{BVI}}{\mathrm{BVI}+\mathrm{FFI}}, \\
& \varphi_{\mathrm{NP}}=\frac{\varphi_{\mathrm{N}} \times \mathrm{FFI}}{\mathrm{BVI}+\mathrm{FFI}},
\end{aligned}
$$

where $\varphi_{\mathrm{NP}}(\mathrm{XJ}-1)=1.29 \%, \varphi_{\mathrm{NP}}(\mathrm{HB}-1)=0.59 \%, \varphi_{\mathrm{NP}}(\mathrm{ZM}-$ $1)=1.03 \%, \varphi_{\mathrm{NP}}(\mathrm{XJ}-2)=1.22 \%, \varphi_{\mathrm{NP}}(\mathrm{HB}-2)=0.40 \%$, and $\varphi_{\mathrm{NP}}(\mathrm{ZM}-2)=0.93 \%$.

\section{Low Temperature Liquid Nitrogen Adsorption Experiment of Pore Characteristics of Coal}

In order to further distinguish the pore type of coal sample and verify the permeability of coal sample, the author utilized the commonly used pore test method, low-temperature liquid nitrogen adsorption experiment, through the analysis of adsorption isotherm and corresponding pore type, combined with nuclear magnetic resonance test data, jointly study the pore structure and permeability of coal.

The experiment used ASAP 2000 specific surface area and pore size distribution instrument to test the nanoscale pore parameters in coal using liquid nitrogen as the adsorption medium. The volume of the adsorption pores in the coal under different pressures was tested at $77.2 \mathrm{~K}$. The relative pressure range was $0.01 \sim 1$, and the test pore size ranged from 0.38 to $260 \mathrm{~nm}$. The analysis method of Чодот et al. $[35,36]$ pore size was used.

According to the adsorption coagulation theory, the adsorption-desorption experiment with capillary pore solids, due to the possible overlap and separation of the adsorption branch and the desorption branch, the adsorption loop will occur. The type of pores is reflected by the shape of the adsorption loop. Coal was a porous medium, and the pore morphology of coal could be further recognized based on the results of adsorption loops. The corresponding pores of the adsorption loop overlap were opened pores at one end, and opened pores at both ends of the adsorption loop were not coincident. The corresponding inflection points in the adsorption loop were ink bottle type and slit type holes [37-41]. The test results of coal sample low temperature liquid nitrogen adsorption were shown in Figure 9.

From the point of view of liquid nitrogen adsorption, the nitrogen adsorption capacity of different coal rank coals was ZM-1 > HB-1 > XJ-1, and the high-order coal liquid nitrogen adsorption amount is up to $8 \mathrm{~cm}^{3} / \mathrm{g}$.

From the pore size distribution curve, in the range of $1 \sim 10 \mathrm{~nm}$, the pore volume corresponding to the ZM-1 coal sample was the largest, reaching $11.8 \times 10^{-4} \mathrm{~cm}^{3} / \mathrm{g}$, and the $\mathrm{XJ}-1$ was the smallest. In $1 \sim 10 \mathrm{~nm}$, the XJ-1 and $\mathrm{HB}$ coal samples increased with the increase of the pore size, and the extreme value of the phase pore volume exhibited a large frequency of oscillation, indicating that there are more pores with different pore sizes in the pore size stage, but the distribution was not uniform. Moreover, the more the micropores of the coal sample, the more the amount of adsorption.
From the type of return line, the liquid nitrogen adsorption curves of XJ-1 and HB-1 coal samples do not coincide, and the coal sample adsorption and desorption curves were relatively flat, indicating that the coal sample contains a large number of cylindrical holes with open ends. The adsorption was only at a relatively large pressure $\left(>0.9 P / P_{0}, P / P_{0}\right.$ is relative pressure), and the adsorption curve rose rapidly, indicating that the proportion of large pores and mesopores in the coal sample was large, and the gas permeability was good. The liquid nitrogen adsorption curve of the ZM-1 coal sample slowly rose, indicating that the coal sample had many micropores. An inflection point occurred at $0.5 P / P_{0}$, indicating that the coal contained an ink bottle type and a slit type hole, and the gas permeability was poor. The results of liquid nitrogen adsorption experiments further confirmed the test of coal magnetic permeability by nuclear magnetic resonance.

\section{Conclusion}

(1) The pores of XJ coal samples developed at all stages, and the connectivity between pores was better; the adsorption pores of $\mathrm{HB}$ and $\mathrm{ZM}$ coal samples were most developed, and the connectivity of the HB coal sample seepage holes was good.

(2) The porosity of the movable fluid of the coal sample was from large to small, respectively. The larger the porosity of the movable fluid of $\mathrm{XJ}>\mathrm{HB}>\mathrm{ZM}$ coal sample, the better the permeability of the coal sample and the more favorable the gas migration in the seepage channel.

(3) Different coal rank coals had different low temperature liquid nitrogen adsorption loops. XJ-1 and HB coals contained more open pores at both ends and better gas permeability, which is beneficial to gas seepage and migration; ZM-1 coal had many micropores, which is beneficial to gas storage; ZM-1 coal pore type was mostly ink bottle type hole, slit type hole, and poor gas permeability.

(4) In general, the nuclear magnetic porosity and permeability of the parallel layered coal samples were better than those of the vertical layered coal samples. Therefore, in the case of mine gas drainage, if the coal seam bedding direction can be considered, the amount of gas extracted may increase and the drainage effect is better.

\section{Data Availability}

The data used to support the findings of this study are included within the article.

\section{Conflicts of Interest}

The authors declare that they have no conflicts of interest.

\section{Acknowledgments}

This work was supported by the National Natural Science Foundation of China (51604101; 51734007; 51704099) and 
funded by China Postdoctoral Science Foundation Grant (2019M652536). This project was also supported by the Henan Postdoctoral Foundation (001801016) and Key R\&D and Promotion Special Projects of Henan Province (192102310511), supported by Zhongyuan Postdoctoral Innovative Talent, and funded by the Doctoral Fund of Henan Polytechnic University (No. B2018-59).

\section{References}

[1] S.-N. Zhou and B.-Q. Lin, The Theory of Gas Flow and Storage in Coal Seams, China Coal Industry Publishing House, Beijing, China, 1999, in Chinese.

[2] H.-L. Liu, H.-Y. Wang, J.-M. Li et al., "Chinese coal bed methane exploitation with clean fuel development mechanism," in Proceedings of the International Symposium on Large-Scale Development of Renewable Energy and Proceedings of the 3rd Pan-Yangtze River Energy Technology Forum, pp. 616-620, Nanjing, China, 2006, in Chinese.

[3] S. Tao, S. D. Chen, and Z. J. Pan, "Current status, challenges, and policy suggestions for coalbed methane industry development in China: a review," Energy Science \& Engineering, vol. 7, no. 4, pp. 1059-1074, 2019.

[4] S. Tao, Z. Pan, S. Chen, and S. Tang, "Coal seam porosity and fracture heterogeneity of marcolithotypes in the Fanzhuang Block, southern Qinshui Basin, China," Journal of Natural Gas Science and Engineering, vol. 66, pp. 148-158, 2019.

[5] D.-J. Zhang, Vigorously Promote Coal Mine Gas Drainage and Utilization, China Emergency Management, Beijing, China, 2009, in Chinese.

[6] D.-J. Zhang, "Vigorously promote coal mine gas drainage and utilization," Journal of Coal Mine Modernization, vol. 1, pp. 1-2, 2010, in Chinese.

[7] S. Tao, Z. J. Pan, S. L. Tang, and S. D. Chen, "Current status and geological conditions for the applicability of CBM drilling technologies in China: a review," International Journal of Coal Geology, vol. 202, pp. 95-108, 2019.

[8] Y.-X. Qi, The Influence of Geological Structure of No. 3 Coal Seam in Xinjing Mine on Coal Pore Structure and Ad-Desorption Characteristics, China University of Mining, Xuzhou, China, 2018, in Chinese.

[9] X. Huang, Experimental Study on the Effect of Microwave Field Action on the Characteristics of Gas Adsorption and Desorption Coal, China University of Mining, Xuzhou, China, 2015, in Chinese.

[10] C.-C. Feng, Experiment Study on Pore Characteristic of Outburst Coal, Liaoning University of Engineering and Technology, Jinzhou, China, 2013, in Chinese.

[11] S. Tao, X. Zhao, D. Z. Tang, C. M. Deng, Q. Meng, and Y. Cui, "A model for characterizing the continuous distribution of gas storing space in low-rank coals," Fuel, vol. 233, pp. 552-557, 2018.

[12] F.-C. Lu, Y.-G. Zhang, and L.-H. Jiang, "Anisotropic characteristics of nuclear magnetic resonance of pores and fractures in coal under uniaxial loading," Coal Geology \& Exploration, vol. 1, pp. 66-72, 2018.

[13] F.-C. Lu, J.-L. Gao, Y.-G. Zhang et al., "Anisotropic ultrasonic characteristics of coal pores and fractures under uniaxial loading," Progress in Geophysics, vol. 6, pp. 2555-2562, 2018, in Chinese.

[14] Y.-B. Yao, D.-M. Liu, D.-Z. Tang et al., "Preservation and deliverability characteristics of coalbed methane, North
China," Petroleum Exploration and Development, vol. 34, no. 6, pp. 664-668, 2007.

[15] Y.-B. Yao, D.-M. Liu, W.-H. Huang et al., "Research on the pore-fractures system properties of coalbed methane reservoirs and recovery in Huainan and Huaibei coalfields," Journal Of China Coal Society, vol. 31, no. 2, pp. 163-168, 2006, in Chinese.

[16] Y.-B. Yao, D.-M. Liu, D.-Z. Tang et al., "Fractal characterization of seepage-pores of coals from China: an investigation on permeability of coals," Computers \& Geosciences, vol. 35, no. 6, pp. 1159-1166, 2009.

[17] E. M. Suuberg, S. C. Deevi, and Y. Yun, "Elastic behaviour of coals studied by mercury porosimetry," Fuel, vol. 74, no. 10, pp. 1522-1530, 1995.

[18] Y.-K. Ma, B.-S. Nie, X.-Q. He et al., "Mechanism investigation on coal and gas outburst: an overview," International Journal of Minerals, Metallurgy and Materials, vol. 1, pp. 1-17, 2020.

[19] Q.-Y. Cheng, B.-X. Huang, and Z.-H. Li, "The research progress in coal or pore and fractures," China Mining Magazine, vol. 21, no. 1, pp. 115-118, 2012, in Chinese.

[20] S.-B. Xie, Y.-B. Yao, J.-Y. Cheng et al., "Research of micropore structure in coal reservoir using low-field NMR," Journal of China Coal Society, vol. 40, no. 1, pp. 170-176, 2015, in Chinese.

[21] Y.-B. Yao, D.-M. Liu, Y.-D. Cai et al., "Fine quantitative characterization of pore fissures of coal based on NMR and X-CT," Scientia Sinica Terrae, vol. 40, no. 11, pp. 1598-1607, 2010.

[22] G.-Q. Zheng, B.-C. Ling, D.-Q. Zheng et al., "The application of nuclear magnetic resonance on analyzing aperture in coal," Journal of North China Institute of Science and Technology, vol. 11, no. 4, pp. 1-7, 2014, in Chinese.

[23] J.-P. Tang and Y.-S. Pan, Nuclear Magnetic Resonance Imaging Theory and Application of Coalbed Methane Occurrence and Migration, Northeastern University Press, Shenyang, China, 2011, in Chinese.

[24] Y. Chen, D. Tang, H. Xu et al., "Pore and fracture characteristics of different rank coals in the eastern margin of the Ordos Basin, China," Journal of Natural Gas Science and Engineering, vol. 26, pp. 1264-1277, 2015.

[25] Y. Sun, C. Zhai, L. Qin, J. Xu, and G. Yu, "Coal pore characteristics at different freezing temperatures under conditions of freezing-thawing cycles," Environmental Earth Science, vol. 77, no. 13, 2018.

[26] J.-P. Tang, H.-N. Tian, N. Yu, and J.-H. Ding, "Experimental study of influence of gas pressure on coal shale gas adsorption characteristics based on nuclear magnetic resonance spectrum," Rock and Soil Mechanics, vol. 37, no. S2, pp. 203-208, 2016, in Chinese.

[27] J.-P. Tang, H.-N. Tian, and Y. Ma, "Experimental study on desorption characteristics of gas in coal shale based on NMR technology," Journal of Liaoning Technical University (Natural Science), vol. 36, no. 3, pp. 282-287, 2017, in Chinese.

[28] L. Luo, Fine Description and Physical Properties of Coal Reservoir in Eastern Junggar Basin, China University of Geosciences, Beijing, China, 2016, in Chinese.

[29] S. Li, D. Tang, H. Xu, and Z. Yang, "The pore-fracture system properties of coalbed methane reservoirs in the Panguan Syncline, Guizhou, China," Geoscience Frontiers, vol. 3, no. 6, pp. 853-862, 2012, in Chinese.

[30] Y. Yao, D. Liu, Y. Che et al., "Petrophysical characterization of coals by low-field nuclear magnetic resonance (NMR)," Fuel, vol. 89, no. 7, pp. 1371-1380, 2010. 
[31] S.-J. Zheng, Y.-B. Yao, Y.-D. Cai et al., "Characteristics of movable fluid and pore size distribution of low rank coals in southern margin of Junggar basin," Coal Geology \& Exploration, vol. 46, no. 1, pp. 56-60, 2018, in Chinese.

[32] Y.-B. Yao, D.-M. Liu, Y. Che et al., "Petrophysical characterization of coals by low-field nuclear magnetic resonance (NMR)," Fuel, vol. 89, pp. 1371-1380, 2017.

[33] Y.-B. Yao, D.-M. Liu, Y.-D. Cai, and J.-Q. Li, "Advanced characterization of pores and fractures in coals by nuclear magnetic resonance and X-ray computed tomography," Science Chian-Earth Sciences, vol. 53, no. 6, pp. 854-862, 2010.

[34] J.-Q. Kang, X.-H. Fu, X. Li, and S. Liang, "Nitrogen injection to enhance methane and water production: an experimental study using the LF-NMR relaxation method," International Journal of Coal Geology, vol. 211, Article ID 103228, 2019.

[35] В. В. Чодот, S.-Z. Song, and Y.-A. Wang, Coal and Gas Outburst, China Industry Press, Beijing, China, 1996.

[36] S. Tao, S. D. Chen, D. Z. Tang, X. Zhao, H. Xu, and S. Li, "Material composition, pore structure and adsorption capacity of low-rank coals around the first coalification jump: a case of eastern Junggar Basin, China," Fuel, vol. 211, pp. 804-815, 2018.

[37] W.-P. Jiang, X.-Z. Song, and L.-W. Zhong, "Research on the pore properties of different coal body structure coals and the effects on gas outburst based on the low-temperature nitrogen adsorption method," Journal of China Coal Society, vol. 36, no. 4, pp. 609-614, 2011, in Chinese.

[38] P. Chen and X.-Y. Tang, "The research on the adsorption of nitrogen in low temperature and micro-pore properties in coal," Journal of China Coal Society, vol. 26, no. 5, pp. 552556, 2001, in Chinese.

[39] J. H. D. Boer, D. H. Everett, and F. S. Stone, The Structure and Properties of Porous Materials, Butterworth, Oxford, UK, 1958.

[40] B.-S. Nie, J.-Y. Lun, K.-D. Wang, and J. Shen, "Three-dimensional characterization of open and closed coal nanopores based on a multi-scale analysis including $\mathrm{CO}_{2}$ adsorption, mercury intrusion, low-temperature nitrogen adsorption, and small-angle X-ray scattering," Energy Science \& Engineering, vol. 8, no. 6, pp. 2086-2099, 2020.

[41] X.-F. Liu, B.-S. Nie, W.-X. Wang, Z. Wang, and L. Zhang, "The use of AFM in quantitative analysis of pore characteristics in coal and coal-bearing shale," Marine and Petroleum Geology, vol. 105, pp. 331-337, 2019. 